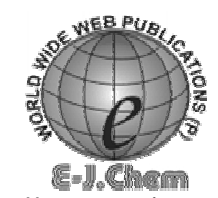

http://www.e-journal net

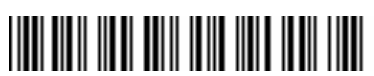

ISSN: 0973-4945; CODEN ECJHAO

E-Journal of Chemistry

Vol. 1, No. 4, pp 189-193, July 2004

\title{
Synthesis of Novel Heterocyclic 4-Thiazolidinone Derivatives and their Antibacterial Activity
}

\author{
K. M. MISTRY and K. R. DESAI* \\ Department of Chemistry \\ South Gujarat University \\ Surat - 395 007, India. \\ k_r_desai@rediffmail.com
}

Received 25 February 2004; Revised \& Accepted 9 June 2004

\begin{abstract}
Thiazolidinones have been prepared by the reaction of various substituted Schiff bases 3 with Thioglycolic acid and Thiolactic acid. The intermediate Schiff bases 3 were synthesized by the condensation of various substituted 2-amino benzothiazole 1 with 1-(4'-methyl Phenyl)-3-methyl-5pyrazolone 2 . The starting compound substituted 2 -amino benzothiazoles were prepared from various substituted amines via substituted phenyl thiourea. The structures of the compounds have been confirmed by elemental analysis and spectral analysis. The antibacterial activity of the compounds has also been screened against Staphylococcus aureus and Escherichia coli.
\end{abstract}

Keywords: Synthesis, Benzothiazole, Schiff Base, 4-Thiazolidinone, Antibacterial activity.

\section{Introduction}

Benzothiazole derivatives were prepared and known to exhibit various biological activities as anti-tuberculotic ${ }^{1}$, anti-allergic ${ }^{2}$. Pyrazole ring system is of some practical importance, because many drugs and medicines contain a pyrazole ring system. As early as 1884 Knorr discovered the antipyretic (temperature reducing) action of a pyrazole derivative in human beings and due to its antipyretic property, he named the compound "Antipyrine". Schiff Base gives good antimicrobial activity and pharmacological applications ${ }^{3}$ and it can be prepared by the acid catalyzed reaction of amines \& ketones or aldehydes. It gives a good fungicidal activity ${ }^{4}$. 4-Thiazolidinones gives good pharmacological properties ${ }^{5}$. 4-Thiazolidinones are known to exhibit antitubercular ${ }^{6}$, antibacterial $^{7}$, anticonvulsant ${ }^{8}$, antifungal ${ }^{9}$, antithyroid activities.

The starting compound substituted 2-amino benzothiazole 1 have been synthesized from various substituted amines ${ }^{10}$. Different substituted 2-amino benzothiazoles were condensed with 1-(4'-methyl Phenyl)-3-methyl-5-pyrazolone to yield Schiff Base 3. The Schiff bases 3 were further reacted with Thioglycolic acid and Thiolactic acid to yield 4-Thiazolidinone derivatives $4 \mathrm{a}-\mathrm{j} \& 5 \mathrm{a}-\mathrm{j}$ respectively. 


\section{Experimental}

All the melting points were determined in open capillary and are uncorrected. The purity of compounds was checked by TLC on silica gel coated glass plates. IR spectra were recorded with $\mathrm{KBr}$ on Shimatzu FT-IR 8300 spectrophotometer, ${ }^{1} \mathrm{H}$ NMR spectra on a Varian Geminy $200 \mathrm{MHz}$ spectrometer using tetramethylsilane as an internal standard.

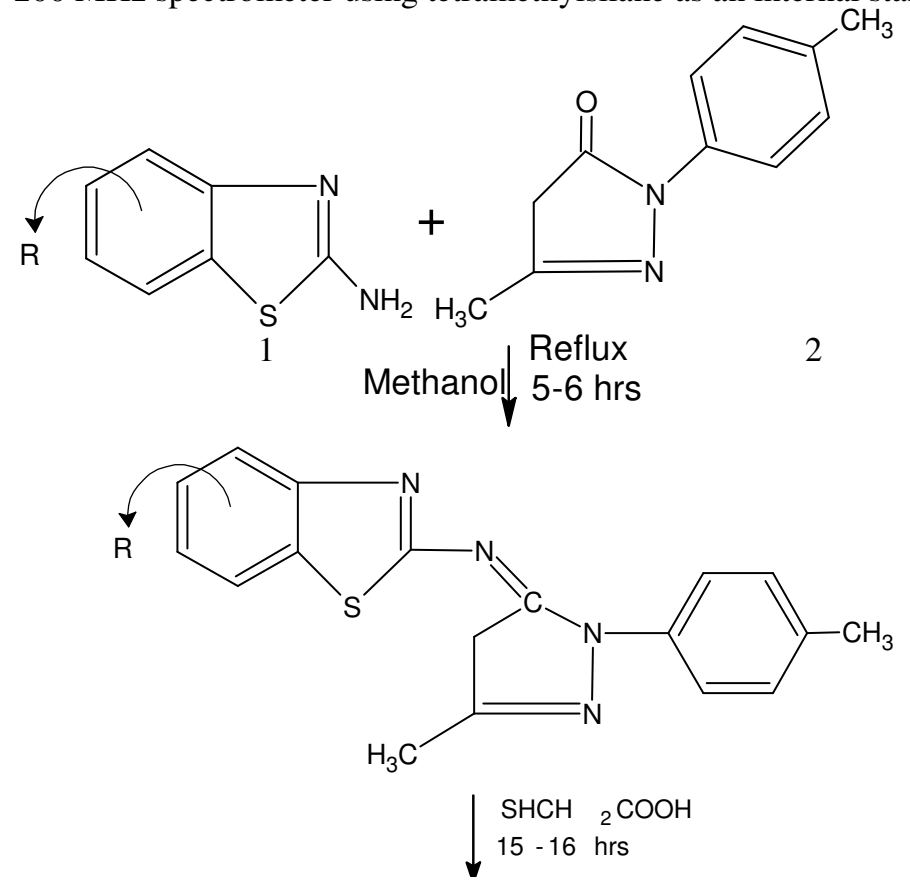

3

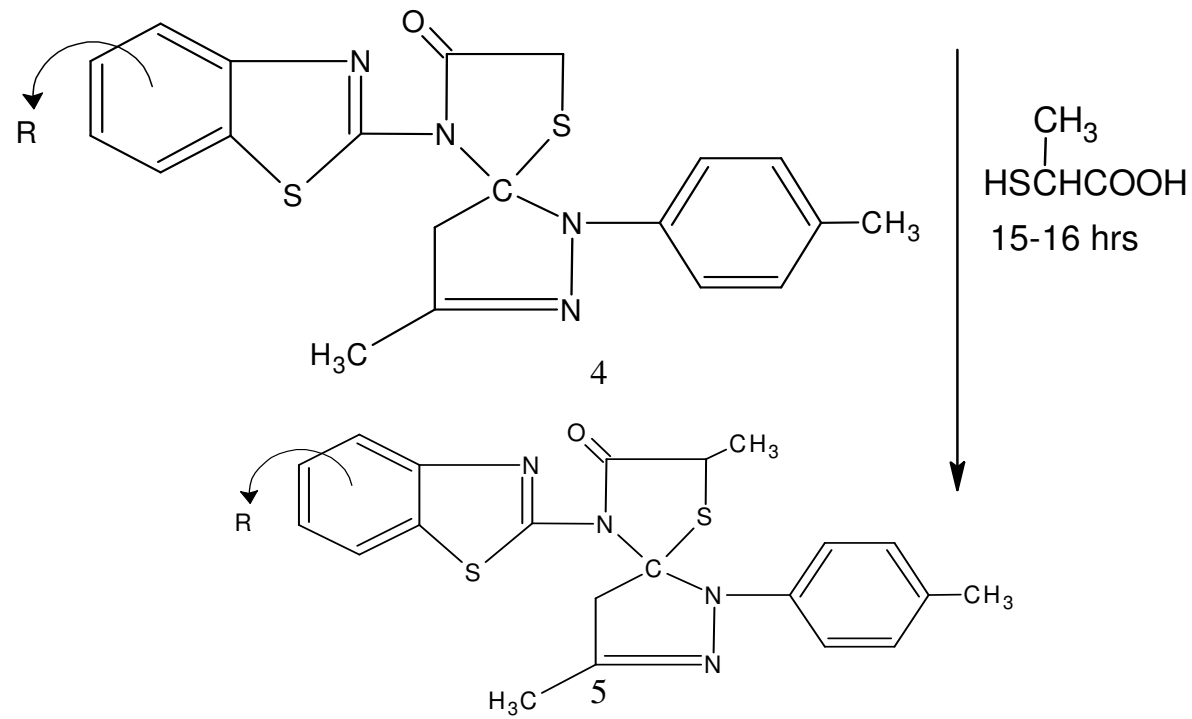

Scheme 1. 
Synthesis of 1-(4'-Methyl phenyl)-3-methyl-5-(2"-imino substituted benzothiazole)pyrazole. (3)

In a $250 \mathrm{~mL}$ R. B. F. mixture of 1-(4'-methyl Phenyl)-3-methyl-5-pyrazolone (0.01 mole) and substituted 2-amino benzothiazole $(0.01$ mole) were taken. About $20 \mathrm{~mL}$ methanol was added to it and refluxed for 5 - 6 hrs. After the completion of reaction, the solvent was removed by vaccum distillation. The solid product was filtered, dried and recrystalised from absolute alcohol. All substituted Schiff bases were prepared in the similar manner.

Synthesis of 2-[spiro-\{1'-(4"-methyl phenyl)-3'-methyl\}-pyrazole]-3-(6"'-nitro benzothiazole)-4-thiazolidinone. (4a)

In a $250 \mathrm{~mL}$ R. B. F. schiff base 3a (0.01 mole, $3.65 \mathrm{~g})$ in benzene was taken, Dean stark apparatus was attached to it and thioglycolic acid $(0.01$ mole, $0.92 \mathrm{~g})$ in benzene was added slowly. Then it was refluxed for $15-16 \mathrm{~h}$, during the course of the reaction the water was removed continuously. The benzene was distilled off to get the thiazolidinone $4 \mathrm{a}$. The solid product was filtered, dried and recrystalised from absolute alcohol. m.p. $145^{\circ} \mathrm{C}$, yield $80 \%$.

The compounds $4 b-j$ were prepared by the same procedure. Their characterization data are shown in Table 1.

Table 1. Characterization data of compounds $4 a-j$

\begin{tabular}{|c|c|c|c|c|c|c|c|}
\hline \multirow[t]{2}{*}{ No. } & \multirow[t]{2}{*}{$\mathrm{R}$} & \multirow[t]{2}{*}{ M.F. (M.W.) } & \multirow[t]{2}{*}{ Yield, \% } & \multirow[t]{2}{*}{ M.P, ${ }^{0} \mathrm{C}$} & \multicolumn{3}{|c|}{$\begin{array}{l}\% \text { Analysis } \\
\text { Calc.(Found) }\end{array}$} \\
\hline & & & & & $\mathrm{C}$ & $\mathrm{H}$ & $\mathrm{N}$ \\
\hline $4 a$ & $6^{\prime \prime \prime}-\mathrm{NO}_{2}$ & $\begin{array}{c}\mathrm{C}_{20} \mathrm{H}_{17} \mathrm{~N}_{5} \mathrm{O}_{3} \mathrm{~S}_{2} \\
(439.0)\end{array}$ & 80 & 145 & $\begin{array}{c}54.66 \\
(54.69)\end{array}$ & $\begin{array}{c}3.87 \\
(3.88)\end{array}$ & $\begin{array}{c}15.94 \\
(15.96)\end{array}$ \\
\hline $4 b$ & $6^{\prime \prime \prime}-\mathrm{SO}_{3} \mathrm{H}$ & $\begin{array}{c}\mathrm{C}_{20} \mathrm{H}_{18} \mathrm{~N}_{4} \mathrm{O}_{4} \mathrm{~S}_{3} \\
(474.0)\end{array}$ & 77 & 165 & $\begin{array}{l}50.63 \\
(50.65)\end{array}$ & $\begin{array}{c}3.79 \\
(3.82)\end{array}$ & $\begin{array}{c}11.81 \\
(11.79)\end{array}$ \\
\hline $4 c$ & $6^{\prime \prime \prime}-\mathrm{CH}_{3}$ & $\begin{array}{c}\mathrm{C}_{21} \mathrm{H}_{20} \mathrm{~N}_{4} \mathrm{OS}_{2} \\
(408.0)\end{array}$ & 75 & 112 & $\begin{array}{l}61.76 \\
(61.73)\end{array}$ & $\begin{array}{c}4.90 \\
(4.87)\end{array}$ & $\begin{array}{c}13.72 \\
(13.75)\end{array}$ \\
\hline $4 d$ & $6^{\prime \prime \prime}-\mathrm{OH}$ & $\begin{array}{c}\mathrm{C}_{20} \mathrm{H}_{18} \mathrm{~N}_{4} \mathrm{O}_{2} \mathrm{~S}_{2} \\
(410.0)\end{array}$ & 76 & 142 & $\begin{array}{l}58.53 \\
(58.52)\end{array}$ & $\begin{array}{c}4.39 \\
(4.43)\end{array}$ & $\begin{array}{l}13.65 \\
(13.68)\end{array}$ \\
\hline $4 \mathrm{e}$ & $4^{\prime \prime \prime}-\mathrm{OCH}_{3}$ & $\begin{array}{c}\mathrm{C}_{21} \mathrm{H}_{20} \mathrm{~N}_{4} \mathrm{O}_{2} \mathrm{~S}_{2} \\
(424.0)\end{array}$ & 72 & 103 & $\begin{array}{c}59.43 \\
(59.46)\end{array}$ & $\begin{array}{c}4.71 \\
(4.73)\end{array}$ & $\begin{array}{c}13.20 \\
(13.24)\end{array}$ \\
\hline $4 \mathrm{f}$ & $6^{\prime \prime \prime}-\mathrm{Cl}$ & $\begin{array}{c}\mathrm{C}_{20} \mathrm{H}_{17} \mathrm{~N}_{4} \mathrm{OS}_{2} \mathrm{Cl} \\
(428.5)\end{array}$ & 77 & 138 & $\begin{array}{c}56.00 \\
(56.04)\end{array}$ & $\begin{array}{c}3.97 \\
(3.95)\end{array}$ & $\begin{array}{l}13.06 \\
(13.09)\end{array}$ \\
\hline $4 \mathrm{~g}$ & $\begin{array}{l}4^{\prime \prime \prime}, 6^{\prime \prime \prime}- \\
\left(\mathrm{NO}_{2}\right)_{2}\end{array}$ & $\begin{array}{c}\mathrm{C}_{20} \mathrm{H}_{16} \mathrm{~N}_{6} \mathrm{O}_{5} \mathrm{~S}_{2} \\
(484.0)\end{array}$ & 68 & 133 & $\begin{array}{c}49.58 \\
(49.61)\end{array}$ & $\begin{array}{c}3.30 \\
(3.29)\end{array}$ & $\begin{array}{l}17.35 \\
(17.36)\end{array}$ \\
\hline $4 \mathrm{~h}$ & $6^{\prime \prime \prime}-\mathrm{OCH}_{3}$ & $\begin{array}{c}\mathrm{C}_{21} \mathrm{H}_{20} \mathrm{~N}_{4} \mathrm{O}_{2} \mathrm{~S}_{2} \\
(424.0)\end{array}$ & 73 & 101 & $\begin{array}{c}59.43 \\
(59.57)\end{array}$ & $\begin{array}{c}4.71 \\
(4.75)\end{array}$ & $\begin{array}{c}13.20 \\
(13.18)\end{array}$ \\
\hline $4 i$ & $4^{\prime \prime \prime}-\mathrm{NO}_{2}$ & $\begin{array}{c}\mathrm{C}_{20} \mathrm{H}_{17} \mathrm{~N}_{5} \mathrm{O}_{3} \mathrm{~S}_{2} \\
(439.0)\end{array}$ & 70 & 118 & $\begin{array}{c}54.66 \\
(54.62)\end{array}$ & $\begin{array}{c}3.87 \\
(3.91)\end{array}$ & $\begin{array}{c}15.94 \\
(15.97)\end{array}$ \\
\hline $4 j$ & $\begin{array}{c}6^{\prime \prime \prime}- \\
\mathrm{NHCOCH}_{3} \\
\end{array}$ & $\begin{array}{c}\mathrm{C}_{22} \mathrm{H}_{21} \mathrm{~N}_{5} \mathrm{O}_{2} \mathrm{~S}_{2} \\
(451.0)\end{array}$ & 71 & 157 & $\begin{array}{r}58.53 \\
(58.55) \\
\end{array}$ & $\begin{array}{c}4.65 \\
(4.67) \\
\end{array}$ & $\begin{array}{c}15.52 \\
(15.50) \\
\end{array}$ \\
\hline
\end{tabular}

Synthesis of 2-[spiro-\{1'-(4"'-methyl phenyl)-3'-methyl\}-pyrazole]-3-(6"'-nitro benzothiazole)-5-methyl-4-thiazolidinone. (5a)

In a $250 \mathrm{~mL}$ R. B. F. schiff base 3a (0.01 mole, $3.65 \mathrm{~g})$ in benzene was taken, Dean stark apparatus was attached to it and thiolactic acid $(0.01$ mole, $1.06 \mathrm{~g})$ in benzene was added slowly. Then it was refluxed for $15-16 \mathrm{hrs}$, during the course of the reaction the water was 
removed continuously. The benzene was distilled off to get the thiazolidinone 5a. The solid product was filtered, dried and recrystalised from absolute alcohol. m.p. $156^{\circ} \mathrm{C}$, yield $72 \%$.

The compounds $5 b-j$ were prepared by the same procedure. Their characterization data are shown in Table 2.

Table 2. Characterization data of compounds $5 \mathrm{a}-\mathrm{j}$

\begin{tabular}{|c|c|c|c|c|c|c|c|}
\hline \multirow[t]{2}{*}{ No. } & \multirow[t]{2}{*}{$\mathrm{R}$} & \multirow{2}{*}{$\begin{array}{l}\text { M.F. } \\
\text { M.W. }\end{array}$} & \multirow{2}{*}{$\begin{array}{c}\text { Yield } \\
\%\end{array}$} & \multirow{2}{*}{$\underset{{ }^{0} \mathrm{C}}{\mathrm{M} . \mathrm{P}}$} & \multicolumn{3}{|c|}{$\begin{array}{l}\% \text { Analysis } \\
\text { Calc.(Found) }\end{array}$} \\
\hline & & & & & $\mathrm{C}$ & $\mathrm{H}$ & $\mathrm{N}$ \\
\hline $5 a$ & $6^{\prime \prime \prime}-\mathrm{NO}_{2}$ & $\begin{array}{c}\mathrm{C}_{21} \mathrm{H}_{19} \mathrm{~N}_{5} \mathrm{O}_{3} \mathrm{~S}_{2} \\
(453.0)\end{array}$ & 72 & 156 & $\begin{array}{c}55.61 \\
(55.64)\end{array}$ & $\begin{array}{c}4.22 \\
(4.25)\end{array}$ & $\begin{array}{c}15.14 \\
(15.10)\end{array}$ \\
\hline $5 b$ & $6^{\prime \prime \prime}-\mathrm{SO}_{3} \mathrm{H}$ & $\begin{array}{c}\mathrm{C}_{21} \mathrm{H}_{20} \mathrm{~N}_{4} \mathrm{O}_{4} \mathrm{~S}_{3} \\
(488.0)\end{array}$ & 71 & 172 & $\begin{array}{l}51.62 \\
(51.64)\end{array}$ & $\begin{array}{c}4.13 \\
(4.16)\end{array}$ & $\begin{array}{l}11.47 \\
(11.45)\end{array}$ \\
\hline $5 \mathrm{c}$ & $6^{\prime \prime \prime}-\mathrm{CH}_{3}$ & $\begin{array}{c}\mathrm{C}_{22} \mathrm{H}_{22} \mathrm{~N}_{4} \mathrm{OS}_{2} \\
(422.0)\end{array}$ & 70 & 127 & $\begin{array}{l}62.53 \\
(62.57)\end{array}$ & $\begin{array}{c}5.25 \\
(5.26)\end{array}$ & $\begin{array}{l}13.26 \\
(13.24)\end{array}$ \\
\hline $5 d$ & $6^{\prime \prime \prime}-\mathrm{OH}$ & $\begin{array}{c}\mathrm{C}_{21} \mathrm{H}_{20} \mathrm{~N}_{4} \mathrm{O}_{2} \mathrm{~S}_{2} \\
(424.0)\end{array}$ & 78 & 158 & $\begin{array}{c}59.41 \\
(59.43)\end{array}$ & $\begin{array}{c}4.75 \\
(4.73)\end{array}$ & $\begin{array}{c}13.20 \\
(13.18)\end{array}$ \\
\hline $5 e$ & $4^{\prime \prime \prime}-\mathrm{OCH}_{3}$ & $\begin{array}{c}\mathrm{C}_{22} \mathrm{H}_{22} \mathrm{~N}_{4} \mathrm{O}_{2} \mathrm{~S}_{2} \\
(438.0)\end{array}$ & 65 & 122 & $\begin{array}{l}60.25 \\
(60.24)\end{array}$ & $\begin{array}{c}5.06 \\
(5.09)\end{array}$ & $\begin{array}{l}12.77 \\
(12.76)\end{array}$ \\
\hline $5 f$ & $6^{\prime \prime \prime}-\mathrm{Cl}$ & $\begin{array}{c}\mathrm{C}_{21} \mathrm{H}_{19} \mathrm{~N}_{4} \mathrm{OS}_{2} \mathrm{Cl} \\
(443.0)\end{array}$ & 70 & 149 & $\begin{array}{c}56.94 \\
(56.97)\end{array}$ & $\begin{array}{c}4.32 \\
(4.36)\end{array}$ & $\begin{array}{l}12.65 \\
(12.67)\end{array}$ \\
\hline $5 g$ & $\begin{array}{l}4^{\prime \prime \prime}, 6^{\prime \prime \prime}- \\
\left(\mathrm{NO}_{2}\right)_{2}\end{array}$ & $\begin{array}{c}\mathrm{C}_{21} \mathrm{H}_{18} \mathrm{~N}_{6} \mathrm{O}_{5} \mathrm{~S}_{2} \\
(498.0)\end{array}$ & 65 & 160 & $\begin{array}{c}50.59 \\
(50.61)\end{array}$ & $\begin{array}{c}3.64 \\
(3.61)\end{array}$ & $\begin{array}{c}16.86 \\
(16.88)\end{array}$ \\
\hline $5 \mathrm{~h}$ & $6^{\prime \prime \prime}-\mathrm{OCH}_{3}$ & $\begin{array}{c}\mathrm{C}_{22} \mathrm{H}_{22} \mathrm{~N}_{4} \mathrm{O}_{2} \mathrm{~S}_{2} \\
(438.0)\end{array}$ & 66 & 113 & $\begin{array}{c}60.25 \\
(60.23)\end{array}$ & $\begin{array}{c}5.06 \\
(5.08)\end{array}$ & $\begin{array}{l}12.77 \\
(12.79)\end{array}$ \\
\hline $5 \mathrm{i}$ & $4^{\prime \prime \prime}-\mathrm{NO}_{2}$ & $\begin{array}{c}\mathrm{C}_{21} \mathrm{H}_{19} \mathrm{~N}_{5} \mathrm{O}_{3} \mathrm{~S}_{2} \\
(453.0)\end{array}$ & 72 & 131 & $\begin{array}{c}55.61 \\
(55.62)\end{array}$ & $\begin{array}{c}4.22 \\
(4.20)\end{array}$ & $\begin{array}{c}15.14 \\
(15.11)\end{array}$ \\
\hline $5 \mathrm{j}$ & $\begin{array}{c}6^{\prime \prime \prime}- \\
\mathrm{NHCOCH}_{3} \\
\end{array}$ & $\begin{array}{c}\mathrm{C}_{23} \mathrm{H}_{23} \mathrm{~N}_{5} \mathrm{O}_{2} \mathrm{~S}_{2} \\
(465.0)\end{array}$ & 64 & 168 & $\begin{array}{r}59.33 \\
(59.31) \\
\end{array}$ & $\begin{array}{r}4.98 \\
(4.95) \\
\end{array}$ & $\begin{array}{c}15.04 \\
(15.06) \\
\end{array}$ \\
\hline
\end{tabular}

\section{Results and Discussion}

Structures of the compounds synthesized have been confirmed by elemental analysis, IR spectra and ${ }^{1} \mathrm{H}$ NMR spectra.

4-Thiazolidinone compound shows IR absorption bands at $1330-1310 \mathrm{~cm}^{-1}\left(\mathrm{Ar}_{-} \mathrm{CH}_{3}\right)$, $800-600 \mathrm{~cm}^{-1}$ (C-S stretching), 1720-1700 $\mathrm{cm}^{-1}$ (C=O stretching) and 1360-1310 $\mathrm{cm}^{-1}$ (C-N stretching), $1690-1640 \mathrm{~cm}^{-1}(\mathrm{C}=\mathrm{N})$.

${ }^{1} \mathrm{H}$ NMR of compound $4 e$

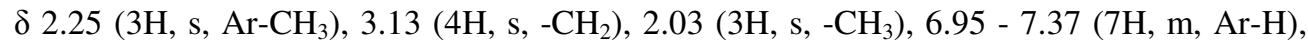
$2.70\left(3 \mathrm{H}, \mathrm{s},-\mathrm{OCH}_{3}\right)$.

\section{${ }^{1} \mathrm{H}$ NMR of compound 5 a}

$\delta 2.3\left(3 \mathrm{H}, \mathrm{s}, \mathrm{Ar}-\mathrm{CH}_{3}\right), 3.08\left(2 \mathrm{H}, \mathrm{s},-\mathrm{CH}_{2}\right), 3.70(1 \mathrm{H}, \mathrm{s},-\mathrm{CH}), 2.10\left(6 \mathrm{H}, \mathrm{s},-\mathrm{CH}_{3}\right), 7.11-7.45$ $(7 \mathrm{H}, \mathrm{m}, \mathrm{Ar}-\mathrm{H})$,

\section{Antibacterial Activity}

The synthesized compounds were tested for their antibacterial activity by measuring the inhibition area on agar plates (diffusimetric method) ${ }^{11}$ with Staphylococcus aureus and Escherichia coli as test germs. 
The results of antibacterial screening indicated that good activity was shown by compounds 4a, 5a, 5h against Staphylococcus aureus and compounds 4j, 5d, 5g, 5j shows good activity towards Escherichia coli. While the compounds 4i, 5d, 5i have less activity against Staphylococcus aureus, and compounds 4f, 5c, 5f have less activity against Escherichia coli. Other compounds showed moderate activity against both bacterial strains. (Table 3)

Table 3. Antibacterial activity of Newly synthesised compounds, zone of inhibition (mm)

\begin{tabular}{cccccc}
\hline No. & S.aureus. & E.coli. & No. & S.aureus. & E.coli. \\
\hline 4a & 12.0 & 9.0 & $5 \mathrm{a}$ & 12.0 & 8.0 \\
4b & 11.0 & 10.0 & $5 \mathrm{~b}$ & 10.0 & 11.0 \\
4c & 9.0 & 8.0 & $5 \mathrm{c}$ & 9.0 & 7.0 \\
4d & 8.0 & 11.0 & $5 \mathrm{~d}$ & 7.0 & 12.0 \\
4e & 8.0 & 10.0 & $5 \mathrm{e}$ & 9.0 & 11.0 \\
4f & 9.0 & 7.0 & $5 \mathrm{f}$ & 11.0 & 7.0 \\
4g & 10.0 & 11.0 & $5 \mathrm{~g}$ & 8.0 & 12.0 \\
4h & 11.0 & 10.0 & $5 \mathrm{~h}$ & 12.0 & 9.0 \\
4i & 7.0 & 8.0 & $5 \mathrm{i}$ & 7.0 & 10.0 \\
4j & 9.0 & 12.0 & $5 \mathrm{j}$ & 10.0 & 12.0 \\
\hline
\end{tabular}

\section{Acknowledgement}

The authors are thankful to the South Gujarat University, Surat for providing research facilities and CDRI, Lucknow for providing ${ }^{1} \mathrm{H}$ NMR spectra.

\section{Reference}

1. Kasel W, Dolezal M, Sidoova E, Odlerova Z and Drsata, J. Chem Abstr., 1989, 110, $128063 \mathrm{e}$.

2. Ronssel U and Jpn Kokai Tokkyo Koho, Chem. Ast.r, 1987, 106, $156494 \mathrm{G}$.

3. Warad D U, Satish C D, Kulkarni V H and Bajgur C S, Indian J. Chem, 2000, 39A, 415.

4. Dash B, Mahapatra P K, Panda D and Patnaik J M, Indian Chem. Soc., 1984, 61, 1061.

5. Yadav R, Srivastava S, Srivastava S K and Srivastava S D, Chemistry An Indian Journal, 2003,1, 95.

6. Desai P S and Desai K R, J. Indian Chem. Soc., 1994, 71, 155.

7. Fadayon M, Kulkarni V D and Pakdaman A S H, Asian J. Chem., 1993, 5(2), 282.

8. Srivastava S K, Srivastava S and Srivastava S D, Indian J. Chem., 1999, 38B, 183.

9. Bhatt J J, Shah B R, Trivedi P B, Undavia N K and Desai N C, Indian J. Chem., 1994, 33B, 189.

10. Ojha K G, Tahiliani H and Jaisinghani N Chemistry An Indian Journal, 2003, 1, 171.

11. Weber A D and Sanders C S, Antimicrob Agents Chemother, 1990, 34, 156. 


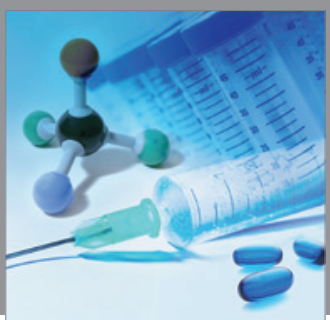

International Journal of

Medicinal Chemistry

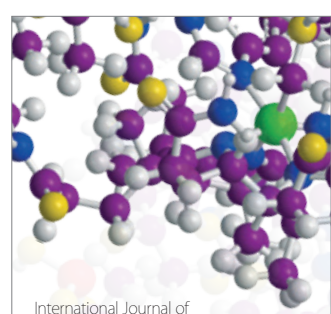

Carbohydrate Chemistry

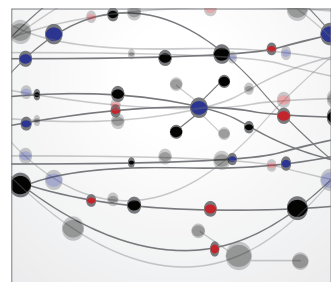

The Scientific World Journal
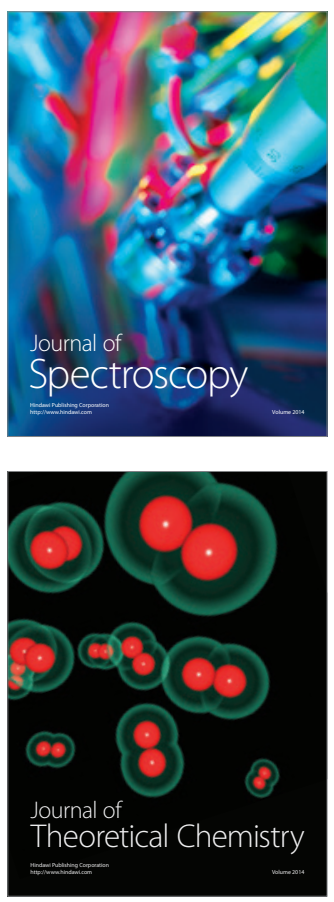
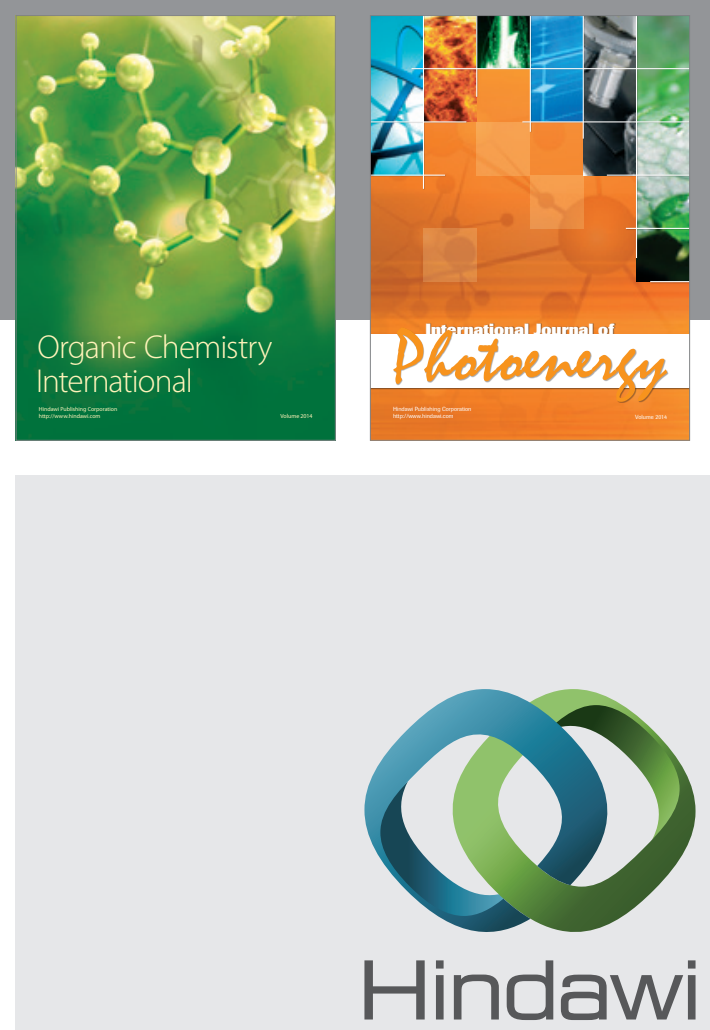

Submit your manuscripts at

http://www.hindawi.com
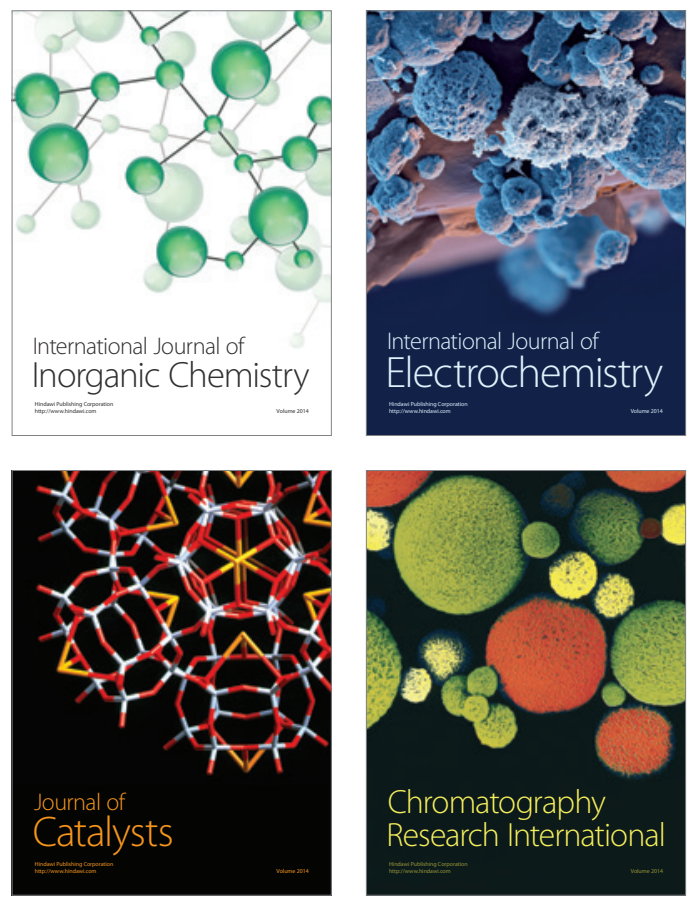
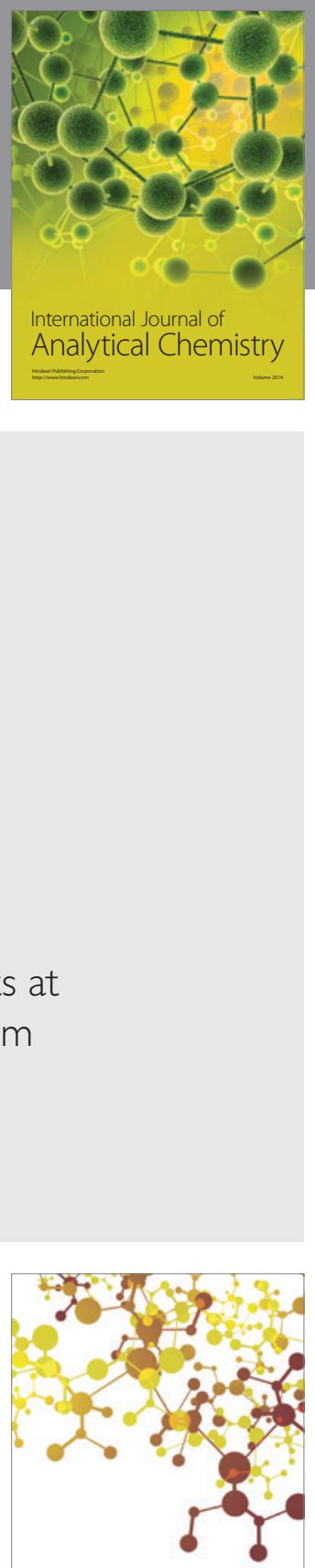

Journal of

Applied Chemistry
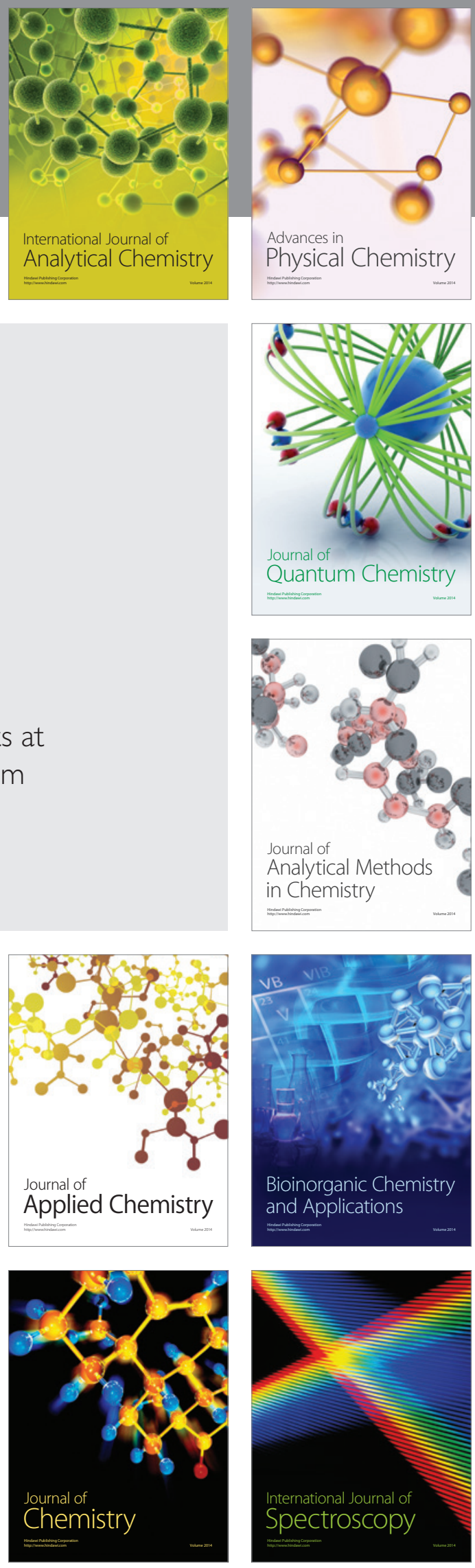